\title{
Clinico-Etiological Profile of New Onset Seizures in Gaeriatric Patients at a Tertiary Care Hospital
}

\author{
Gurram Rajasekhar Reddy ${ }^{1}$, Vishvanayak ${ }^{2}$ \\ ${ }^{1}$ Post Graduate Student, Department of Medicine, Teerthanker Mahaveer Medical College \& Research Centre, Moradabad, ${ }^{2}$ Professor, Department of \\ Medicine, Teerthanker Mahaveer Medical College \& Research Centre, Moradabad.
}

\section{Abstract}

Background: Seizure is defined as "a paroxysmal event due to abnormal excessive or synchronous neuronal activity in the brain." Based on discharge characteristics and its distribution, this unusual activity can manifest either as focal or generalized tonic clonic to absence seizures which are not easily noticeable by observer. For 2010, as indicated by the Global Burden of Disease (GBD) investigation, epilepsy accounted for $0.7 \%$ of burden globally i.e, more than 17 million DALYs. Studies conducted in India have reported variable incidence rates which varies from 0.2 to 0.6 per 1,000 population. The incidence rates reported from India are similar to most of the developed countries and less than most of the developing nations which ranged from 1.0 to 1.9 per 1,000 per year. AIM: The aim of the study is to assess \& identify the various clinico-etiological factors causing first onset of seizures in gaeriatric patients. Rationale: Several studies are available on seizure in elderly in western population but very few studies are done in this part of the world. Etiological profile of seizures varies from place to place because of difference in socio-economic and cultural attributes, prevalence of various diseases in a particular area and several other factors. This study aims to observe the clinico-etiological profile of new onset seizures in elderly in Moradabad region of western Uttar Pradesh. Subjects and Methods: This study was carried out at Teerthanker Mahaveer Medical College and Research Centre, Moradabad (UP) as an observational hospital based study. 51 patients of new onset seizures above 60 years of age. Inclusion Criteria: All patients with new onset seizures and were more than 60 years of age. Exclusion Criteria: All patients with age less than 60 years. All known cases of Seizure disorder/Epilepsy were excluded. All those who did not give consent. Results: Out of 51 elderly patients, males accounted for $55 \%$, females accounted for $45 \%$ and a gender ratio of 1.2:1 for male to female. Among 51 patients with new-onset seizures, hypertension was seen in $29.4 \%$ patients as co-morbidity and $13.7 \%$ patients had both hypertension and diabetes as co-morbidities. The study also showed that generalized tonic-clonic seizures (GTCS) were found among $76.5 \%$ cases whereas among $23.5 \%$ patients were of focal seizures. However, acute and remote symptomatic were almost found in an equal proportion $47.1 \%$ and $45.2 \%$ respectively and only $7.8 \%$ were idiopathic. It was noted that the maximum number of patients had CVA as the cause of new onset seizures in this age group, of which $90.9 \%$ patients had an ischaemic stroke. 14 patients had metabolic insult of which, $35.71 \%$ patients had uremic encephalopathy as the cause of new onset seizures. $60 \%$ of seizures in CNS infections are secondary to tuberculosis probably because of high burden of tuberculosis in the study area. Conclusion: In our study CVA was the frequently observed etiology of seizures among study population and the second most common was metabolic insults. Among stroke, seizures occured more commonly in ischemic stroke than in hemorrhagic stroke. In our study GTCS was the most common type of seizure in $76.5 \%$ of study population. And acute symptomatic and remote symptomatic were almost seen in equal numbers $47.1 \%$ and $45.2 \%$ respectively. CVA accounted for most of the remote symptomatic seizures of which ischemic stroke is most common and metabolic causes accounted for most of the acute symptomatic seizures. The high burden of tuberculosis in the study area may have accounted for this higher percentage (60\%) of seizures secondary to tuberculosis in the study patients under CNS infections sub group.

Keywords: Seizures, Epilepsy, Geriatric Population.

Corresponding Author: Dr. Vishvanayak, Professor, Department of Medicine, Teerthanker Mahaveer Medical College \& Research Centre, Moradabad.

Received: December 2019

Accepted: December 2019

\section{Introduction}

A seizure is defined as "a transient occurrence of signs and/or symptoms due to abnormal excessive or synchronous neuronal activity in the brain. Depending on the distribution of discharges, this abnormal brain activity can have various manifestations, ranging from dramatic convulsive activity to experiential phenomena not readily discernible by an observer". ${ }^{[1]}$ Epilepsy is described "as a condition in which a person has recurrent seizures due to a chronic underlying process". [1]

Epidemiological studies have showed that the incidence and prevalence of seizure is highest in the elderly patients. India, the second-most populous country in the world, has experienced a dramatic demographic transition during the past 50 years, with a significantly increasing population above the age of 60 years. Thus, among patients with seizures, elderly individuals constitute the fastest growing segment. New onset seizure in elderly patients poses diagnostic difficulties when compared to seizures occurring in younger individuals. This may relate to difficulties of 
acquiring precise medical history, atypical presentations and frequent association with various comorbidities, which all complicate the clinical profile of seizures in the gaeriatric age groups. ${ }^{[2]}$

Around $16.50 \%$ of Indian population is accommodated by the largest state of India, Uttar Pradesh in which few studies were carried out to assess the clinico-etiological profile among gaeriatric patients. ${ }^{[3]}$ The etiology of seizures also varies according to geographic distribution and the cultural attributes of the area. For example, in some states due to easy availability of alcohol, alcohol related seizures are more prevalent. Therefore this study is done with the intention of studying the clinical and etiological profile of seizures in gaeriatric patients pertaining to area of Western Uttar Pradesh, India. The emphasis is on seizures beginning in the elderly.

For 2010, as indicated by the Global Burden of Disease (GBD) investigation, epilepsy accounted for $0.7 \%$ of burden globally i.e, more than 17 million DALYs. Among the south East Asia regions, the epilepsy prevalence is about 2 to 10 per 1,000 population and India accounted for significant proportion of the total DALYs (Disability Adjusted Life Years). ${ }^{[4]}$

Based on clinical features and EEG findings the ILAE has released recent guidelines on approach to seizure classification.

\section{Classicationof Seizures}

1. Focal seizure (Can be further described as having cognitive, motor, autonomic, sensory, or other features)

2. Generalized seizures

a. Motor

- Tonic clonic

- Other motor(e.g., atonic, myoclonic)

b. Non motor (absence)

3. Unknown onset

(Can be further described as motor or non-motor, or unclassified)"

\section{$\underline{\text { AIM }}$}

- The aim of the study is to assess \& identify the various clinico-etiological factors causing first onset of seizures in gaeriatric patients

\section{Objectives}

- To determine clinico-etiological profile of new onset seizures among gaeriatric patients using a step wise approach at Teerthanker Mahaveer Medical College and Research Centre

\section{Subjects and Methods}

\section{Study design \& Place}

This study was carried out at Teerthanker Mahaveer Medical College and Research Centre, Moradabad (UP) as an observational hospital based study.

\section{Study Population}

All elderly patients of age more than 60 years with new onset seizures who presented during the study period of one year at Teerthanker Mahaveer Medical College \& Hospital.

\section{Period of Study}

The period of study was of 12 months. The thesis protocol was approved by both ethical and scientific committees of TMMC \& RC.

\section{Sample Size}

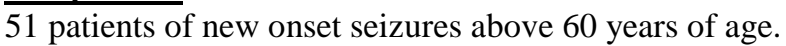

\section{Inclusion Criteria}

- All patients with new onset seizures and were more than 60 years of age.

\section{Exclusion Criteria}

- All patients with age less than 60 years.

- All known cases of Seizure disorder/Epilepsy were excluded.

- All those who did not give consent.

\section{Data collection technique and tools}

- All the patients with new onset seizure after 60 years were recruited during the study phase.

- Prior to the data collection Institutional Ethical Committee approval was obtained.

- Information regarding patients past medical history, detailed description of seizure event and the sequence of events which lead to seizure, neurological findings, and treatment if any, were noted. This information was recorded in a proforma.

- Seizure type was established based on 2017 ILAE classification system of seizures.

- Investigations

The following investigations were done in the patients included.

- Serum glucose, Electrolytes, LFT, RFT.

- EEG and Neuroimaging studies were advised for all patients.

- X-ray chest, ultrasound abdomen, 2D echo, ECG, Thyroid tests based on necessity.

\section{Results}

\section{Data Analysis and Interpretation}

A total of 51 patients gave consent and were included in our study; of them $55 \%$ (28 patients) were male individuals and $45 \%$ (23 patients) were female individuals. The age of patients distributed between 60 to 90 years age and mean of study population was 68 years (+_7.14SD). All the cases involved in this study had first episode of seizure after 60 years of age. Every patient was assessed clinically and by routine investigations and special investigations where indicated.

The demographic distribution and various characteristics of seizure and of the patients included in the study are as discussed below. 
Table 1: Age wise distribution of patients Sample Characteristics

Age in years (mean+_SD) $=68$

years(+_7.14 SD)

\begin{tabular}{|c|c|c|}
\hline & $\mathbf{f}$ & $\%$ \\
\hline 60-75 Years & 44 & 86.3 \\
\hline 76-90 Years & 07 & 13.7 \\
\hline
\end{tabular}

In our study, $86.3 \%$ (44 patients) of study population belonged to $60-75$ years age group and $13.7 \%$ ( 7 patients) of study population belonged to 75-90 years age group and mean age of the study population is calculated as 68 years(+_7.14SD).

Table 2: Gender wise distribution of patients

Sample Characteristics

Gender

\begin{tabular}{|l|} 
Male \\
\hline Female \\
\hline
\end{tabular}

\begin{tabular}{|l|l|}
\hline $\mathbf{f}$ & $\mathbf{\%}$ \\
\hline 28 & 55.0 \\
\hline 23 & 45.0 \\
\hline
\end{tabular}

As seen in the above table, in our study $55 \%$ (28 patients) of study population were males and $45 \%$ (23 patients) of study population were females.

Table 3: Table showing distribution of patients based on Types of Seizures

\begin{tabular}{|l|l|l|l|}
\hline Sample Characteristics & f & \% \\
\hline \multirow{2}{*}{ Types of Seizures } & GTCS & 39 & 76.5 \\
\cline { 2 - 4 } & Focal Seizure & 12 & 23.5 \\
\hline
\end{tabular}

In our study, Generalized tonic clonic seizure (GTCS) was observed in $76.5 \%$ (39 patients) of study population. Focal seizure was observed in $23.5 \%$ (12patients). Overall, the most frequently observed seizure type in study population is GTCS.

\begin{tabular}{l} 
Table 4: Distribution According to Etiology of Seizure in \\
Elderly (N=51) \\
\begin{tabular}{|l|l|}
\hline ETIOLOGY & f $(\%)$ \\
\hline 1.CVA (n=22) & $\mathbf{2 2}$ \\
\hline Ischaemic stroke & $20(90.90 \%)$ \\
\hline Subdural hematoma & $1(4.54 \%)$ \\
\hline ICH (Intra Cerebral Haemorrhage) & $1(4.54 \%)$ \\
\hline 2.Metabolic (n=14) & $\mathbf{1 4}$ \\
\hline Hyponatremia & $1(7.14 \%)$ \\
\hline Hypoglycaemia & $4(28.57 \%)$ \\
\hline Uremic encephalopathy & $5(35.71 \%)$ \\
\hline Co2 narcosis/hyperglycaemia & $1(7.14 \%)$ \\
\hline Septic encephalopathy & $3(21.42 \%)$ \\
\hline 3.CNS Infections (n=5) & $\mathbf{5}$ \\
\hline Tuberculomas & $2(40 \%)$ \\
\hline TBM \&vasculitic infarct & $1(20 \%)$ \\
\hline Neurocystiscercosis & $1(20 \%)$ \\
\hline Cryptococcal meningitis & $1(20 \%)$ \\
\hline 4.CNS Tumour $(\mathbf{n}=\mathbf{4})$ & $\mathbf{4}$ \\
\hline Calcified granuloma & $1(25 \%)$ \\
\hline Meningioma & $1(25 \%)$ \\
\hline Metastasis & $2(50 \%)$ \\
\hline $\mathbf{5 . O t h e r s ~ ( n = 2 ) ~}$ & $\mathbf{2}$ \\
\hline Neuro degenerative disorder & $1(50 \%)$ \\
\hline Post traumatic seizures & $1(50 \%)$ \\
\hline 6. Idiopathic(n=4) & $\mathbf{4}$ \\
\hline & \\
\hline &
\end{tabular} \\
\hline
\end{tabular}

From the above table, it is illustrated that the $43.1 \%$ (22 patients) of study population had Cerebrovascular Disease being the most common cause, $27.4 \%$ (14 patients) of study population had metabolic causes and second most common cause observed in study population.
Out of 22 patients with CVA, ischemic stroke was seen in 90.9\% (20 patients) and hemorrhagic stroke in $4.54 \%$ (1 patient) and subdural hematoma in $4.54 \%$ (1 patient).

Out of 14 patients with metabolic insults as cause of seizures, $35.71 \%$ (5 patients) had uremic encephalopathy, $28.57 \%$ (4 patients) had hypoglycemia and $21.42 \%$ (3 patients)had septic encephalopathy and in $7.13 \%$ (1 patient), hyponatremia and $\mathrm{CO}_{2}$ narcosis is observed which resulted in new onset seizures in study population.

Next to metabolic are CNS infections which are seen in 5 patients, of which tuberculosis accounts for $60 \%$ (3 patients) of cases and neurocysticercosis and cryptococcal meningitis $20 \%$ (1 patient) each.

CNS tumour accounted for 4cases, of which $50 \%$ (2 patients) had metastasis.

Other causes like neurodegenerative and post traumatic seizures are observed in 1 patient each. No cause was identified in 4 patients.

Table 5: Association between types of seizures with etiology of seizures $(\mathbf{N}=\mathbf{5 1})$

\begin{tabular}{|l|l|l|}
\hline \multirow{2}{*}{ Etiology of Seizures } & \multicolumn{2}{|l|}{ Types of Seizures } \\
\cline { 2 - 3 } & GTCS $(\mathbf{n = 3 9})$ & Focal Seizures $(\mathbf{n = 1 2})$ \\
\hline Stroke & $12(54.5 \%)$ & $10(45.5 \%)$ \\
\hline Metabolic & $14(100 \%)$ & $0(0 \%)$ \\
\hline CNS Infections & $4(80 \%)$ & $1(20 \%)$ \\
\hline CNS Tumour & $3(75 \%)$ & $1(25 \%)$ \\
\hline Idiopathic & $4(100 \%)$ & $0(0 \%)$ \\
\hline Others & $2(100 \%)$ & $0(0 \%)$ \\
\hline
\end{tabular}

In our study, as described in the above table, GTCS is the most frequently observed seizure type in all the etiological subgroups.100\%(14 patients) of metabolic insults patients and $54.5 \%$ (12 patients) of stroke patients had GTCS and accounted for significant proportion of this seizure type. Focal seizures occurred in 12 patients and more commonly observed in stroke patients which accounted for $83.3 \%$ of focal seizure patients.

Table 9: Association between types of seizures based on etiology with various causes of seizures $(\mathrm{N}=51)$

\begin{tabular}{|l|l|l|l|}
\hline \multirow{2}{*}{ Etiology of Seizures } & \multicolumn{3}{|l|}{ Types of Seizures } \\
\cline { 2 - 4 } & $\begin{array}{l}\text { Acute } \\
\text { Symptomatic } \\
(\mathbf{n = 2 4 )}\end{array}$ & $\begin{array}{l}\text { Remote } \\
\text { Symptomatic } \\
(\mathbf{n = 2 3 )}\end{array}$ & $\begin{array}{l}\text { Idiopathic } \\
(\mathbf{n = 4})\end{array}$ \\
\hline Stroke & $4(16.7 \%)$ & $18(78.3 \%)$ & $0(0 \%)$ \\
\hline Metabolic & $14(58.3 \%$ & $0(0 \%)$ & $0(0 \%)$ \\
\hline CNS Infections & $5(20.8 \%)$ & $0(0 \%)$ & $0(0 \%)$ \\
\hline CNS Tumour & $0(0 \%)$ & $4(17.4 \%)$ & $0(0 \%)$ \\
\hline Idiopathic & $0(0 \%)$ & $0(0 \%)$ & $4(100 \%)$ \\
\hline Others & $1(4.2 \%)$ & $1(4.3 \%)$ & $0(0 \%)$ \\
\hline
\end{tabular}

In our study, as described in the above table it is observed that acute symptomatic is observed in 24 patients and remote symptomatic observed in 23 patients. Most of remote symptomatic seizures occurred due to stroke in $78.3 \%$ (18 patients) while most of acute symptomatic seizures were due to metabolic causes in $58.3 \%$ (14 patients) of cases.

\section{Discussion}

An observational study was conducted among elderly 
patients of age more than 60 years with new-onset seizures during the one year study period at TMMC\&RC, located in Moradabad, India. The study population comprised of a total of 51 elderly patients with first episode of seizure occuring after 60 years of age. The seizure type was established based on 2017 ILAE classification system of seizures. All the patients involved in the study had first episode of seizure after 60 years of age. In this era, proper history, clinical examination, appropriate investigations and analysis of etiology are required to reduce associated morbidity and mortality in elderly new-onset seizures and its association with different causes.

\section{Age \\ In the present study out of 51 patients, age of $86.3 \%$ patients was between $60-75$ years, and of $13.7 \%$ aged $76-90$ years. In our study, the average age when the first seizure occurred was 68 years (+_7.14SD). The study findings were similar to the findings reported by Hussain et al. and in their study they observed that incidence of seizures is more in 60-74 age group when compared to 75-89 age group. ${ }^{[5]}$ Another study conducted by Wallace showed similar results that the seizure incidence increases with age in 65-80 years age group. ${ }^{6]}$}

\section{Gender}

The present study showed that among 51 patients of newonset seizures, $55 \%$ were males while $45 \%$ were females. In our study males were predominant and this was similar to results reported that by Muralidhar and Venugopal, Hirani and Shrivastava, and Sendil et al. ${ }^{[7-9]}$

\section{Co-morbidities}

In this study, a total of 27 patients with comorbidities were observed. $29.4 \%$ of study population had hypertension as comorbidity, $9.8 \%$ of study population had diabetes and $13.7 \%$ of study population had both hypertension and diabetes as co-morbidities. Hypertension was the most common comorbidity in our study followed by diabetes mellitus which is similar to several studies. ${ }^{[10]}$ A study conducted by Chen and Wang showed that diabetes mellitus was associated with late-onset of seizures. ${ }^{[11]}$

\section{Type of seizures}

In our study, it is observed that GTCS was the most frequently observed seizure type seen in $76.5 \%$ of study population whereas in $23.5 \%$ it is focal in onset. GTCS was the most common seizure type observed in our study. Similar results were revealed by Narayanan and Murthy, Kanitkar et al., Sendil et al., and Hirani and Shrivastava also reported that maximum number of patients were of GTCS seizure type in adults $(55 \%, 70 \%, 64 \%$, and $60 \%$, respectively). ${ }^{[8,9,12,13]}$

In this study, $47.1 \%$ of study population had acute symptomatic seizures. whereas in the study by Sander et al, ${ }^{[14]}$ and Sinha et al, ${ }^{[15]}$ it was $57.8 \%$ and $15 \%$ respectively . Acute symptomatic seizures occur at the time of systemic insult or in close temporal association with a documented brain insult. Seizures are considered to be acute symptomatic if they occur in the first 7 days of illness.

In the present study, remote symptomatic seizures accounted for $45.2 \%$ of study population. In the study by Sander et al, ${ }^{[14]}$ and Sinha et al, ${ }^{[15]}$ it accounted for $21 \%$ and $18.8 \%$ of cases respectively. It is observed that metabolic causes accounted for $58.3 \%$ of acute symptomatic seizures and stroke accounted for $75 \%$ of remote symptomatic seizures. This was similar to Assis et al. result, ${ }^{[16]}$ who also reported that ischemic stroke $(37 \%)$ was more common than hemorrhagic stroke $(12 \%)$. The occurrence of late seizures is most probably secondary to underlying anomalies like scarring, alterations in excitability of neurons \& hemosiderin deposition. ${ }^{[17]}$ Overall, in this study acute symptomatic and remote symptomatic were almost found in equal numbers $47.1 \%$ and $45.2 \%$ respectively.

\section{Etiology of seizures}

The various causes responsible for seizures among the patients included is as described in [Table 4]. From the table, it is observed that $43.1 \%$ had Cerebrovascular disease; followed by $27.4 \%$ patients had metabolic causes. In CVA patients, ischemic stroke was seen in $90.9 \%$ and hemorrhagic stroke in $4.54 \%$ and sub dural hematoma $4.54 \%$ patients as the cause of new onset seizures in elderly. In metabolic causes $35.71 \%$ had uremic encephalopathy, $28.57 \%$ had hypoglycemia, $21.42 \%$ had septic encephalopathy and hyponatremia $\& \mathrm{CO}_{2}$ narcosis in $7.13 \%$ each as reason for new onset seizure in elderly. In this study among stroke, ischemic stroke was the most frequent cause. Whereas among metabolic causes it was uremic encephalopathy. These results were comparable to study done by Kanitkar et al. ${ }^{[12]}$ who reported that stroke and metabolic cause as the first and second most common cause of seizures in $44 \%$ and $26 \%$ respectively.

In our study, the third most common cause observed was secondary to CNS infections of which tuberculosis accounted for $60 \%$ of cases and neurocysticercosis and cryptococcal meningitis in $20 \%$ each. CNS tumour accounted for $7.8 \%$, idiopathic causes in $5.8 \%$ and neurodegenerative causes in $1.9 \%$ and post traumatic seizure noted in $1.9 \%$ of study population as the cause of new onset seizures in elderly.

Uttar pradesh being one the high burden areas of tuberculosis in india may have accounted for this higher percentage $(60 \%)$ of patients having seizures secondary to tuberculosis under CNS infections sub group.

The results were similar to study conducted by Ravi Prakash Pandey et al, in which the most common cause of seizure was stroke $(56.86 \%)$ followed by metabolic causes $(13.72 \%) .{ }^{[18]}$ In a study conducted by K. S.Amaravathi et al, ${ }^{[19]}$ and Sownthariya et al, ${ }^{[20]}$ at a tertiary care hospital, the most common aetiology of seizures was Stroke, constituting to $38 \%$ of cases. Our results were contrasted with the studies conducted by Pradeep et al., Jiménez et al., Sendil et al., and Hirani and Shrivastava that showed most common underlying etiology was idiopathic $(33.9 \%)$ followed by metabolic $(20.3 \%)$, CNS infections $(16.9 \%)$, and stroke $(10.2 \%)$ in their studies. Our study also, contrast the findings of the study conducted by Quraishi et al. which stated that the most common etiologies of generalized seizures among elderly age-groups were CNS infections and stroke $(29.7 \%$ each), followed by idiopathic (27\%), metabolic (10.8\%), and tumors $(2.7 \%) .^{[8,9,21]}$ 
GTCS is the most common seizure type observed in study population. Among the GTCS patients, metabolic causes were most common and seen in $35.9 \%$ patients followed by a stroke in $30.7 \%$ patients with GTCS. Meanwhile, stroke has accounted for maximum number of cases of focal seizures i.e., $83.3 \%$.

In this study, the association between types of seizures and EEG findings it was found that abnormal EEG was observed in maximum number of cases with GTCS i.e, $69.2 \%$, and normal electroencephalography was seen $30.8 \%$ patients. And all the patients (100\%) with focal seizures have shown abnormal electroencephalography.

\section{Conclusion}

The study was conducted in a particular region of the rural population of Moradabad district of Uttar Pradesh where there is substantial burden of seizures with large rural population and limited health care resources which require proper counseling, diagnostic modalities and treatment.

In our study CVA was the frequently observed etiology of seizures among study population and the second most common was metabolic insults. Among stroke, seizures occured more commonly in ischemic stroke than in hemorrhagic stroke. In our study GTCS was the most common type of seizure in $76.5 \%$ of study population. And acute symptomatic and remote symptomatic were almost seen in equal numbers $47.1 \%$ and $45.2 \%$ respectively. CVA accounted for most of the remote symptomatic seizures of which ischemic stroke is most common and metabolic causes accounted for most of the acute symptomatic seizures. The high burden of tuberculosis in the study area may have accounted for this higher percentage $(60 \%)$ of seizures secondary to tuberculosis in the study patients under CNS infections sub group.

\section{References}

1. Fisher, R. S., Boas, W. van E., Blume, W., Elger, C., Genton, P., Lee, P., \& Engel, J. (2005). Epileptic Seizures and Epilepsy: Definitions Proposed by the International League Against Epilepsy (ILAE) and the International Bureau for Epilepsy (IBE). Epilepsia, 46(4), 470-472. doi:10.1111/j.0013-9580.2005.66104.x

2. Verma, A., \& Kumar, A. (2016). Clinical and etiological profile of epilepsy in elderly: a hospital-based study from rural India. Acta Neurologica Belgica, 117(1), 139-144.

3. Amudhan, S., Gururaj, G., \&Satishchandra, P. (2015). Epilepsy in India I: Epidemiology and public health. Annals of Indian Academy of Neurology, 18(3), 263-277.
4. Reddy VM, Chandrashekar CR. Prevalence of mental and behavioural disorders in India : A meta-analysis. Indian J Psychiatry 1998;40:14957.

5. Wallace H, Shorvon S, Tallis R. Age-specific incidence and prevalence rates of treated epilepsy in an unselected population of 2052922 and age-specific fertility rates of women with epilepsy. Lancet 1998;352:1790-3.

6. Muralidhar V, Venugopal K. New onset seizures: Etiology and corelation of clinical features with computerized tomography and electroencephalography. J Sci Soc. 2015;42:82-7. [Google Scholar].

7. Hirani MM, Shrivastva S. Clinical profile of new onset seizures in adults. Indian J Appl Res. 2015;5:19-21. [Google Scholar].

8. Sendil G, Kumar AN, Kumar MV. Late onset shake-etiology at stake A prospective study. Int J Sci Stud. 2014;2:20-4. [Google Scholar]Yang, PJ, Poul EB, Michael EC, Patncia KD. Computed tomography and childhood seizure disorders. Neurology 1979; 29: 10888.

9. Chen Yun and Wang Xuefeng, Association Between Seizures and Diabetes Mellitus: A Comprehensive Review of Literature, Current Diabetes Reviews (2013) 9: 350 .

10. Menon B, Shorvon SD (2009) Ischaemic stroke in adults andepilepsy. Epilepsy Res 87:1-11.

11. Narayanan T, Murthy JM. New onset acute symptomatic seizures in a neurological Intensive Care Unit. Neurol India. 2007;55:136-40. [PubMed] [Google Scholar].

12. Kanitkar SA, Gaikwad AN, Kalyan M, Aarwal R, Krunal K, Tamakuwala KK, et al. Study of seizure disorder in elderly: Etiology, types, EEG and image findings. Transworld Med J. 2013;1:24-5. [Google Scholar].

13. Assis TR, Bacellar A, Costa G et al (2015) Etiological prevalence of epilepsy and epileptic seizures in hospitalized elderly in a Brazilian tertiary center-Salvador-Brazil. ArqNeuropsiquiatr 73(2):83-89

14. Study of Epilepsy: newly Sander JWAS, Hart YM,Johnson AL, ShorvonSD.National General Practice diagnosed epileptic seizures in a general population. Lancet. 1990;336:1267-1271.

15. Sinha S.Satish Chandra P, Kalband BR, ThennarasuK, New-onset status epilepticus and cluster seizures in the elderly, J Cin Neurosci.2013;20:423-8

16. Assis TR, Bacellar A, Costa G et al (2015) Etiological prevalence of epilepsy and epileptic seizures in hospitalized elderly in a Brazilian tertiary center- Salvador-Brazil. ArqNeuropsiquiatr 73(2):83-89.

17. Doria JW, Forgacs PB. Incidence, Implications, and Management of Seizures Following Ischemic and Hemorrhagic Stroke. Springer Link 2019. Available at: https://dx.doi.org/10.1007/s11910-019-0957-4 (accessed November 28, 2019).

18. Ravi Prakash Pandey, Anurag Chaurasia, Sunil Ahuja, PanchalingppaBetageri, Manoj Indurka. A Study of Clinical Profile of Seizure Disorder in Geriatric Population, Sch. J. App. Med. Sci., 2017;5:237-243.

19. Amaravathi KS, Nagamani R, Sakuntala P, Shyamsunder MN, Rajasekhar PV, Gopalakrishna V. A Study on Clinical Profile of New Onset Focal Seizures in a Tertiary Care Centre. International Journal of Scientific and Research Publications. 2015 Jul.

20. R. Sownthariya, Heber Anandan. Study of EEG abnormalities in migraine. International Journal of Contemporary Medical Research 2017;4:1743-1744.

21. Menon B, Shorvon SD (2009) Ischaemic stroke in adults and epilepsy. Epilepsy Res 87:1-11.

Copyright: (c) the author(s), 2019. It is an open-access article distributed under the terms of the Creative Commons Attribution License (CC BY 4.0), which permits authors to retain ownership of the copyright for their content, and allow anyone to download, reuse, reprint, modify, distribute and/or copy the content as long as the original authors and source are cited.

How to cite this article: Reddy GR, Vishvanayak. Clinico-Etiological Profile of New Onset Seizures in Gaeriatric Patients at a Tertiary Care Hospital.Acad. J Med. 2019;2(2):195-99.

DOI: dx.doi.org/10.21276/ajm.2019.2.2.50

Source of Support: Nil, Conflict of Interest: None declared. 